\title{
Linkage analysis
}

National Cancer Institute ( $\mathrm{NCl})$

\section{Source}

National Cancer Institute (NCI). Linkage analysis.

A gene-hunting technique that traces patterns of disease in high-risk families. It attempts to locate a disease-causing gene by identifying genetic markers of known chromosomal location that are co-inherited with the trait of interest. 